\title{
Near-Infrared SERS Nanoprobes with Plasmonic Au/Ag Hollow-Shell Assemblies for In Vivo Multiplex Detection
}

\author{
Homan Kang ${ }^{1}$, Sinyoung Jeong ${ }^{2}$, Yoon-Sik Lee ${ }^{1}$, Ho-young Lee ${ }^{3}$, and Dae Hong Jeong ${ }^{2}$ \\ ${ }^{1}$ School of Chemical and Biological Engineering, Seoul National University, Seoul 151-742, Republic of Korea \\ ${ }^{2}$ Department of Chemistry Education, Seoul National University, Seoul 151-742, Republic of Korea \\ ${ }^{3}$ Department of Nuclear Medicine, College of Medicine, Seoul National University, Seoul 151-742, Republic of Korea \\ (jeongdh@snu.ac.kr)
}

\begin{abstract}
Nanostructures of noble metal such as gold and silver exhibit an optical phenomenon known as surface-enhanced Raman scattering (SERS), which enhances Raman scattering of molecules adsorbed on the surface of them. When SERS is used as a coding method, it has advantages for bioassays over other optical tools: A narrow spectral band of SERS peaks (less than $0.5 \mathrm{~nm}$ ), which is able to make a large number of coded, flexible selectivity of photoexcitation line covering UV to NIR region, and no-photobleaching feature. [1-5]. Owing to these advantages, SERS active bio-probe can be utilized for multiplexed detection of numerous bio-targets. Nevertheless, SERS nanoprobes still need to be improved in sensitivity and tissue penetration ability for in vivo SERS application. In this regard, novel metal nanoshells have been extensively studied to prepare the SERS active nanoparticles because of their high surface area and unique optical properties. In particular, hollow Au/Ag nanostructures have tunable extinction band from visible to near infrared (NIR) region (ca. 400-1200 nm), exhibiting both surface plasmon resonance and scattering [6-8].

In this study, we introduce the preparation of plasmonic $\mathrm{Au} / \mathrm{Ag}$ hollow-shell assemblies (Au/Ag-HSA) as high sensitive SERS active nanoprobes in NIR region. The Au/Ag nanoshells were formed on the surface of silica sphere from silver nanoparticles embedded silica sphere via galvanic replacement reaction. Raman reporter molecules were then absorbed on the $\mathrm{Au} / \mathrm{Ag}$ surface, and subsequently encapsulated with silica shell to prevent dissociation of label compounds and aggregation of probes. The Au/Ag-HSA exhibited highly sensitive signals due to their localized surface electromagnetic field and the assembled multiple $\mathrm{Au} / \mathrm{Ag}$ nanoshells on silica sphere. Further it shows extinction (absorption/scattering) in NIR region, the transparent window of biological tissue. These results show that Au/Ag-HSA can be applied to in vivo optical imaging as a SERS nanoprobe.
\end{abstract}

\section{References}

1. William E. Doering and Shuming Nie: Anal. Chem. 75 (2003) 6171.

2. Jeremy D. Driskell, Karen M. Kwarta, Robert J. Lipert, and Marc D. Porter: Anal. Chem. 77 (2005) 6147.

3. Xing Su, Jingwu Zhang, Lei Sun, Tae-Woong Koo, Selena Chan, Narayan Sundararajan, Mineo Yamakawa, and Andrew A. Berlin: Nano Lett. 5 (2005) 49.

4. Musundi B. Wabuyele and Tuan Vo-Dinh: Anal. Chem. 77 (2005) 7810.

5. Bong-Hyun Jun, Gunsung Kim, Mi Suk Noh, Homan Kang, Yong-Kweon Kim, Myung-Haing Cho, Dae Hong Jeong, and Yoon-Sik Lee: Nanomedicine 6 (2011) 1463.

6. Y. Sun, B. Mayers, Y. Xia: Adv. Mater. 15 (2003) 641.

7. R. Weissleder: Nat. Biotechnol. 19 (2001) 316.

8. Sangyeop Lee, Hyangah Chon, Moonkwon Lee, Jaebum Choo, Soon Young Shin, Young Han Lee, Im Joo Rhyu, Sang Wook Son, and Chil Hwan Oh: Biosensors and Bioelectronics 24 (2009) 2260. 